\title{
Litiasis urinaria. Presente y futuro
}

\author{
J.A. Lancina Martín \\ Coordinador Nacional de Litiasis de la Asociación Española de Urología (2000-2004).
}

Actas Urol Esp 2005; 29 (4): 339-344

Y a se han cumplido más de veinte años desde que se introdujera en la práctica clínica el uso de la litotricia extracorpórea por ondas de choque (LEOC). Efectivamente esta modalidad técnica, que revolucionó el tratamiento de la litiasis urinaria, ya ha cumplido su mayoría de edad. Lejos quedan aquellas noticias que nos venían llegando desde el Departamento de Urología de la Universidad de Munich, en donde Chaussy y su equipo estaban investigando un procedimiento capaz de fragmentar los cálculos urinarios mediante la aplicación de una energía externa, que era transmitida a través del propio organismo sin ocasionar daño tisular, mediante la colocación de un acoplador sobre la superficie cutánea. Aún recuerdo la incredulidad inicial de algunos colegas cuando empezaron a llegar las primeras informaciones de los buenos resultados conseguidos con el nuevo método en los primeros pacientes tratados a comienzos del año 1980. Esta sorpresa inicial estaba más que justificada porque la tecnología médica de entonces apenas hacía presagiar que pudiera diseñarse un prototipo de estas características y que fuera seguido de tan buenos resultados. En cierto modo, este descubrimiento se adelantó a su época y tuvo una trascendencia tal que fue más allá del ámbito de la propia Urología e incluso del propio estamento medico, haciéndose eco la noticia en todos los medios de comunicación del mundo.

Pero aquellos años 80 no significaron solamente la introducción de la LEOC en el tratamiento de la litiasis urinaria sino que, en un tiempo ligeramente anterior, ya se habían incorporado las llamadas técnicas de cirugía mínimamente invasiva (CMI) como la nefrolitotomía percutánea (NLP), difundida por Alken y Wickham de forma independiente, y la ureterorrenoscopia (URS), difundida por Pérez-Castro. Todo ello contribuyó a un cambio radical en la forma de tratar la litiasis. Hasta entonces sólo era posible la cirugía abierta tradicional, que había alcanzado un grado de desarrollo sorprendente. La técnica quirúrgica, con aportaciones como la pielolitotomía ampliada de Gil-Vernet y la nefrolitotomía anatrófica de Smith y Boyce, permitía abordar con éxito litiasis complejas de gran masa litiásica. También supusieron avances técnicos notables los métodos de preservación de la función renal (hipotermia, inosina intraarterial, etc.) para minimizar los efectos de la isquemia durante el clampaje de la arteria renal, los métodos de localización intraoperatoria de fragmentos litiásicos (radiología de contacto, ecografía, nefroscopia, etc.) y la aplicación de ingeniosos sistemas de extracción de los fragmentos alojados en cálices inaccesibles (como la pielolitotomía por coágulo de Dees). Todos estos avances consiguieron que pudieran ser tratados quirúrgicamente la mayoría de los cálculos, por muy complejos que fueran, respetando en lo posible el principio de conservación del parénquima y el funcionalismo renal, sin que por ello se descuidase el objetivo de conseguir la total eliminación del cálculo. Este objetivo se hacía especialmente muy necesario en la litiasis coraliforme infectiva si se quería evitar la recidiva, el crecimiento de fragmentos residuales o la misma persistencia de la infección urinaria. En el postoperatorio siempre existía la posibilidad de disolver los cálculos residuales mediante la irrigación de sustancias quimiolíticas a través de una sonda de nefrostomía dejada al final de la intervención. Pues bien, cuando estaba perfectamente establecido el tratamiento quirúrgico de la litiasis urinaria, de forma repentina se tuvo que cambiar el modo de tratar los cálculos con el advenimiento de la CMI y la LEOC.

Desde que en el año de 1984 se introdujo el litotriptor extracorpóreo Dornier HM3 (conocido popularmente como la bañera por la necesidad de tener que sumergir al paciente en un contenedor lleno de agua) realmente pocos cambios se 
han producido en la tecnología que se hayan traducido en una mejoría significativa de los resultados. De hecho, el modelo HM3 se sigue considerando el gold-standard al que deben seguir comparándose los nuevos prototipos que salen al mercado. Los cambios producidos en los litotriptores, llamados de segunda y después tercera generación, han ido más bien en la dirección de conseguir equipos más fácilmente manejables para los médicos y más confortables para los pacientes, prescindir de la anestesia, costes de adquisición y mantenimiento inferiores, uso más restringido de materiales desechables y menores necesidades de espacio para su instalación y de mantenimiento. Es indudable que todos estos cambios han producido mejoras funcionales en las Unidades de Litotricia, pero este hecho no ha repercutido necesariamente en unos mejores resultados en cuanto a limpieza de cálculos. Más bien al contrario, los nuevos equipos consiguen resultados inferiores al primitivo Dornier HM3. Las limitaciones que este modelo tenía para el tratamiento de la litiasis ureteral fueron superadas por los litotriptores de segunda generación. Con la introducción del Lithostar de Siemens y el Dornier MFL 5000 fue posible tratar todos los cálculos, independientemente de su localización, mejorando notablemente la manejabilidad técnica y además sin tener que sumergir en agua al paciente aunque, sin embargo, todo ello a costa de conseguir unos resultados inferiores a los obtenidos previamente.

De los sistemas que inicialmente se propusieron para la generación y transmisión de las ondas de choque: el sistema electrohidráulico (que incorporó el HM3 de Dornier), el electromagnético (que incorporó el Lithostar de Siemens) y el piezoeléctrico (que incorporó el LTO1 de Edap), el desarrollo de los acontecimientos ha derivado en el triunfo del sistema electromagnético, a tal punto que ha sido adoptado por la misma Dornier en sus nuevos prototipos. El éxito de este sistema se debe a la mayor sencillez de manejo, mayor duración de los componentes, buena energía en punto focal para conseguir fragmentación adecuada, posibilidad de integración fácil en equipos multifuncionales y menores necesidades de mantenimiento. El sistema electrohidráulico precisa del cambio frecuente de electrodos y su manejo y mantenimiento son más complejos. El sistema piezoeléctrico tiene los peores resultados de eficacia litofragmentadora, con mucha necesidad de retratamientos, aunque es el sistema que ocasiona menos dolor. Por todas estas consideraciones, la mayoría de los fabricantes se inclinan actualmente por el diseño de equipos con sistema electromagnético.

Un inconveniente de los nuevos litotriptores es la menor calidad de imagen de los sistemas de localización del cálculo. Efectivamente, tanto el sistema de localización radiológica como ecográfica, precisan de una calidad de imagen muy alta para permitir una adecuada focalización para la emisión de las ondas de choque. El éxito del tratamiento reside en gran parte en conseguir este objetivo. La carrera de bajada de precios, que han seguido las distintas compañías en el diseño de nuevos litotriptores, ha derivado en una menor calidad de los sistemas de localización, lo que ha reducido las prestaciones y, consecuentemente, los resultados respecto a los primeros litotriptores.

Por otra parte, desde la aparición de los litotriptores de segunda generación se propuso prescindir de la anestesia porque los nuevos prototipos eran pretendidamente indoloros. Como consecuencia de esta nueva situación, las Unidades de Litotricia, donde inicialmente se realizaban anestesias por ser imprescindibles con la HM3, comenzaron a desplazar a los anestesiólogos. El tiempo ha venido a demostrar lo equivocado de esta nueva orientación. El disconfort que se produce en la mayoría de los pacientes durante el tratamiento ha obligado a introducir técnicas de sedo-analgesia intravenosa que, en muchos casos, es asumida por los mismos urólogos responsables de la litotricia. Un planteamiento que debemos hacernos en el futuro inmediato es la necesidad de que cada Unidad de Litotricia cuente permanentemente con anestesista para el soporte de la analgesia del paciente. Por muy simples que sean las medidas para controlar el dolor, esta responsabilidad debe ser asumida por el anestesista y no por el urólogo. En la distribución actual de tareas para la atención integral del paciente, que la dinámica hospitalaria impone, implica que el anestesiólogo sea el responsable del control del dolor. Indudablemente esto va a suponer un aumento de los costes del procedimiento. 
El litotriptor ideal sería aquel que pudiera alcanzar el nivel de potencia necesario en el punto focal para conseguir la fragmentación del cálculo en partículas lo más diminutas posibles para facilitar su eliminación, sin por ello producir daño tisular, reduciendo en lo posible la necesidad de retratamientos, que tenga un manejo sencillo y que disponga de un buen sistema de localización del cálculo, que sea confortable para el paciente y no requiera de la aplicación de anestesia, que no suponga altos costes en adquisición ni programas de mantenimientos frecuentes y un uso restringido de materiales desechables, con tasas altas de limpieza de cálculos y mínimas complicaciones. Este litotriptor aún no está disponible, pero debemos confiar en que las investigaciones puestas en marcha conduzcan en los próximos años al diseño de equipos que se aproximen a las características comentadas.

La CMI tuvo un gran desarrollo en la Urología como consecuencia de su aplicación precisamente en la litiasis urinaria. La NLP y la URS supondrán el primer intento con éxito de la incorporación de las técnicas de mínima invasión en la Urología. Su introducción fue en tiempo anterior a la LEOC y, antes de que ésta fuera una realidad, estás técnicas tuvieron una extraordinaria difusión, desplazando en buena parte a la cirugía convencional. La llegada de la LEOC supuso un brusco frenazo para el desarrollo de estas técnicas. Poco tiempo después se puso de manifiesto que la LEOC tenía limitaciones para la resolución de determinado tipo de cálculos, ya fuera por su gran tamaño, por su extraordinaria dureza o por la dificultad de eliminación de los fragmentos a consecuencia de una anatomía desfavorable.

La facilidad que suponía administrar la LEOC redujo radicalmente las indicaciones de la $\mathrm{CMI}$ aunque, en muchos casos, no encontraba debida justificación. Las litiasis complejas no podían ser resueltas completamente con la LEOC o, en el mejor de los casos, lo conseguía después de aplicar múltiples sesiones, con lo que se prolongaba excesivamente el tiempo de resolución. Si bien la URS compitió con la LEOC en los cálculos de uréter pelviano, la NLP vio disminuidas sus indicaciones a la mínima expresión. Sin embargo, en el momento actual existe una tendencia a aumentar las indicaciones de estas técnicas en perjuicio de la LEOC.
Por otra parte, se pudo comprobar desde el principio el beneficio de la utilización de varios procedimientos para la resolución de casos complejos. Así, el uso de la NLP y la LEOC en la litiasis coraliforme conseguía mejorar los resultados que cada técnica conseguía individualmente, con una aceptable morbilidad. También en ciertas complicaciones de la LEOC, como la calle-litiásica ureteral, podía aplicarse la URS u otras técnicas endourológicas con finalidad desobstructiva, como la nefrostomía percutánea o el cateterismo ureteral. La colocación de un catéter doble J previamente a la LEOC en pacientes con cálculos voluminosos podía minimizar los efectos de la impactación de los fragmentos litiásicos en uréter durante su eliminación.

La NLP apenas ha sufrido evolución desde los inicios de la técnica. La dilatación del trayecto percutáneo, clásicamente realizada con los dilatadores coaxiales telescopados de Alken o los dilatadores teflonados de Amplatz, va ganando adeptos en el uso de los balones de dilatación, que permiten en un solo tiempo dilataciones de hasta un calibre de $30 \mathrm{Fr}$. Otra novedad ha sido la introducción de la técnica minipercutánea (mini-perc) consistente en el uso de mininefroscopios de $18 \mathrm{Fr}$ que permiten su introducción a través de una vaina de Amplatz de 20 Fr. Esta técnica puede encontrar algunas indicaciones cuando sea posible minimizar el acceso percutáneo. La posibilidad de introducir instrumentos flexibles por el abordaje percutáneo ha mejorado las expectativas de eliminación de cálculos caliciales de difícil acceso. No se ha extendido mucho el uso de varios accesos percutáneos en una misma sesión quirúrgica debido a la invasividad del procedimiento. Tampoco ha sido bien considerado el acceso por el cáliz superior debido al elevado riesgo de lesión pleural.

Los instrumentos utilizados para la URS han experimentado continuas evoluciones en sus diseños, desde los primitivos ureteroscopios rígidos de grueso calibre ( $14 \mathrm{Fr}$ ) hasta el miniureteroscopio semirrígido de Dretler (7,5 Fr), con el fin de facilitar el avance del instrumento a través del uréter sin necesidad, en muchas ocasiones, de dilatar el orificio meatal y ni siquiera precisar de anestesia. La miniaturización de los ureteroscopios se ha visto beneficiada por la incorporación de nuevas fuentes de energía externa para la 
litotricia intracorpórea, en particular el láser, cuyas finas fibras pasan fácilmente por los canales de trabajo de estos ureteroscopios. Otro cambio notable ha sido la introducción de ureteroscopios flexibles con una visión aceptable y manejo cómodo, permitiendo un avance fácil por todo el uréter e incluso llegar hasta las cavidades renales. Esta nueva posibilidad técnica nos permite eliminar cálculos caliciales de difícil acceso percutáneo.

Donde se han producido más novedades ha sido en las fuentes de energía para litotricia intracorpórea. Desde las primeras fuentes disponibles de litotricia electrohidráulica, abandonada en parte por la posibilidad de lesión tisular, y los ultrasonidos, se consiguió un gran avance con la introducción del láser colorante Candela que se ha visto superado por el láser de holmium. Este último láser necesita poco tiempo para su puesta a punto y tiene un mantenimiento relativamente sencillo, utiliza una fina fibra flexible que puede ser introducida por finos instrumentos, rígidos o flexibles, consiguiendo un poder de fragmentación muy elevado sin producir apenas desplazamiento de los cálculos. Su mayor inconveniente es el elevado coste de adquisición. En el momento actual, el láser holmium supone la fuente de energía de elección para la litiasis ureteral.

La aparición de la litotricia neumática (Swiss Lithoclast) también supuso un avance notable por la extraordinaria sencillez en su manejo, precisando de pocos recursos para su puesta en funcionamiento (solamente el aire comprimido del quirófano), sin necesidad de piezas desechables y bajo coste de adquisición. Su mayor inconveniente radica en el desplazamiento que provoca sobre el cálculo con cada pulsación, lo que ha limitado su aplicación en la litiasis ureteral. Sin embargo, en la actualidad se considera, junto a la litotricia ultrasónica, como la fuente de energía de primera elección para la litiasis renal y vesical. Posteriormente han ido apareciendo nuevas fuentes de energía, algunas de gran utilidad como la litotricia electrocinética, pero que aún no han conseguido extenderse demasiado.

La LEOC sigue siendo el tratamiento de elección para la litiasis renal, suponiendo aproximadamente el 80-85\% de las indicaciones iniciales. En general, los cálculos de tamaño inferior a 2 $\mathrm{cm}$ son una buena indicación para la LEOC. Los cálculos de mayor tamaño, en particular los cálculos coraliformes y aquellos que por razones anatómicas tienen evidentes dificultades en la eliminación de los fragmentos (ángulo infundíbulopélvico inferior reducido, estrechez infundibular, anomalías anatómicas, cambios postquirúrgicos de la vía, etc.) deben ser tratados mediante NLP. La cirugia abierta debe ser reservada para menos del $5 \%$ de los casos.

Antes de decidir un tratamiento con LEOC en una litiasis compleja debemos plantearnos dos cuestiones fundamentales: qué previsión tendremos de que exista una litiasis residual y cuántas sesiones creemos que precisará para su resolución. Evidentemente no siempre es fácil conocer de antemano la respuesta a estas dos preguntas, pero en muchos casos podemos hacer una estimación. En aquellos pacientes que se prevea una litiasis residual superior al 30\% y aquellos que precisen de más de 3 sesiones para su resolución, debería ser cuestionada la indicación de una LEOC como tratamiento único.

En algunos casos será preciso el tratamiento combinado de diversos procedimientos. Unos serán planteados de forma estratégica, en particular cálculos renales de tamaño grande o coraliformes, en donde se puede comenzar con una cirugía percutánea y, para aquellos cálculos alojados en cálices no accesibles a la percutánea, se puede aplicar la LEOC en un segundo tiempo. También, de forma alternativa, se puede aplicar la URS transuretral con ureteroscopio flexible y láser de holmium, particularmente en cálculos alojados en cálices inferiores. En otras ocasiones el fracaso de una LEOC inicial, con litiasis residual significativa, deberá seguir de una cirugía percutánea o incluso URS transuretral, dependiendo de las características de cada caso.

Para la litiasis ureteral, la LEOC sigue siendo el tratamiento de elección para la gran mayoría de los cálculos localizados en uréter superior, siempre que su tamaño no exceda de $2 \mathrm{~cm}$. En cálculos de mayor tamaño se impone la URS, en particular si tienen carácter obstructivo. Existe una mayor controversia para los cálculos localizados en uréter inferior, donde la LEOC y la URS compiten por ser el tratamiento de elección. Si bien la URS es la indicación apropiada para cálculos de tamaño superior a $2 \mathrm{~cm}$, la discusión se produce para cálculos de menor tamaño. En este 
tipo de cálculos, ambos procedimientos son igualmente eficaces con un porcentaje de éxitos superior al $80-90 \%$. Con la URS se consigue la resolución con una sola sesión quirúrgica en la mayoría de los pacientes, mientras que con la LEOC hasta en un 25\% de los casos necesita más de una sesión, lo que prolonga el tiempo de resolución del cálculo. Sin embargo, la URS precisa de hospitalización y del uso de anestesia, aunque en algunos casos pueden ser evitadas (particularmente en mujeres). Las complicaciones, aunque reducidas en ambos procedimientos, tienen una mayor relevancia en la URS. No está claro tampoco la ventaja de un procedimiento sobre otro en términos de coste-efectividad. Para aquellas unidades que cuenten con todo el armamentario terapéutico, la LEOC puede ser más coste-efectiva.

La CMI está ganando protagonismo en el tratamiento de la litiasis urinaria en los últimos años, y es posible que esta tendencia se consolide en el futuro. Esta situación viene derivada de los fracasos que se obtienen con la LEOC en determinado tipo de cálculos (consistencia dura, voluminosos, coraliformes, asociados a malformaciones o cambios postquirúrgicos severos), en donde la litiasis residual supera al 30\% de los casos. Es por ello que la tendencia actual es favorable a la aplicación de técnicas quirúrgicas desde el principio en cálculos con estas características, reservando a la LEOC como un tratamiento secundario ante una posible litiasis residual. La falta en la progresión de los nuevos litotriptores, que pudieran mejorar los resultados históricos conseguidos hasta el momento, junto al avance producido en el diseño de los instrumentos endoscópicos y la incorporación de nuevas fuentes de energia para litotricia intracorpórea, han posibilitado mayores indicaciones para la CMI.

Los cálculos de consistencia más dura (oxalato cálcico monohirato, brushita y cistina) ofrecen más resistencia a la LEOC, incluso aunque tengan un pequeño tamaño, y, por lo tanto, con frecuencia serán indicaciones para la CMI. Los cálculos de ácido úrico puro deberán ser sometidos inicialmente a tratamiento médico disolvente pues se consigue su disolución en la mayoría de los casos. Cuando estos cálculos alcanzan un gran tamaño o cuando está asociada otra composición, pueden precisar de la aplicación de LEOC para facilitar su eliminación, a la vez que permite exponer más superficie del cálculo para favorecer el efecto del tratamiento disolvente.

La progresión imparable de la laparoscopia en el campo de la Urología ha podido encontrar también indicaciones para la litiasis urinaria, aunque posiblemente con menor impacto que en otras patologías. La razón de este hecho debemos encontrarla en que la laparoscopia no entra en competición con la cirugía abierta en la litiasis, como sucede en otras parcelas, sino que lo hace directamente con la NLP, la URS y la LEOC, todas ellas consideradas técnicas mínimamente invasivas como la misma laparoscopia. No obstante, se vislumbran en el futuro inmediato indicaciones de la laparoscopia en la litiasis que pueden desplazar a la NLP y URS. Con la NLP puede entrar en dura competición en los cálculos piélicos, en particular cuando están alojados en pelvis amplias o extrasinusales, porque así se evitaría el daño sobre el parénquima renal durante la dilatación del trayecto percutáneo. Otra indicación posible de la laparoscopia serían los cálculos alojados en divertículos caliciales. Contrariamente la laparoscopia tendrá mayores dificultades para competir con la URS, aunque puede ser una buena indicación en cálculos de uréter superior con un tamaño superior a $2 \mathrm{~cm}$ o en donde ya ha fracasado la LEOC.

La cirugía abierta debe quedar relegada al mínimo (no más del 5\% de los casos) cuando tenemos a nuestra disposición todos los procedimientos terapéuticos. Efectivamente aún quedan algunos casos en que la cirugía abierta puede estar indicada como en litiasis complejas (cálculos de gran volumen, grandes malformaciones, alteraciones severas de la vía urinaria, etc.) y, por supuesto, cuando fracase la LEOC y la CMI. La laparoscopia puede competir con algunas de las pocas indicaciones que aún quedan para la cirugía abierta, como por ejemplo la nefrectomía parcial en litiasis calicial inferior con foco litógeno y atrofia parenquimatosa.

El tratamiento quimiolítico por irrigación directa de sustancias a través de catéteres de nefrostomía o ureterales solamente es posible para cálculos compuestos de estruvita, ácido úrico o cistina, pero esta modalidad terapéutica está actualmente en desuso debido a que exige 
hospitalizaciones largas, provocan gran disconfort a los pacientes y existe posibilidad de complicaciones infecciosas y de toxicidad sistémica. Por ser una técnica costosa, molesta y fácilmente sustituible por la LEOC o CMI, la quimiólisis por irrigación tienen un reducido campo de aplicación en la actualidad.

La litiasis vesical debe ser abordada por extracción endoscópica transuretral, o percutánea transvesical en algunos casos, donde también será necesaria, la mayoría de las veces, la resolución conjunta del proceso obstructivo (HBP, esclerosis cervical, etc.) que es la causa de su formación o el motivo que impide su eliminación. Cuando el factor causal precise de cirugía abierta (por ejemplo HBP de gran tamaño), la litiasis será extraída en el mismo acto quirúrgico abierto. La LEOC puede estar indicada en cálculos no excesivamente grandes y en donde, además, no esté presente factor causal orgánico.

A pesar de los mensajes que se han venido repitiendo en los últimos años acerca del potencial de recidiva de la litiasis (superior al 50\% a largo plazo) y la posibilidad de reducir esta tasa de recidivas, incluso con simples medidas profilácticas, el urólogo sigue mostrando cierta resistencia al estudio de los factores de riesgo litogénico y, por ende, de la adopción de medidas activas de prevención de la recidiva.

En los últimos años se han producido notables avances en el estudio de la influencia que tienen algunos factores en la formación de los cálculos urinarios como el rol de factores genéticos, la influencia de algunos componentes de la dieta y de otros factores medio-ambientales. Los estudios prospectivos del grupo de Curhan en la Universidad de Harvard, siguiendo poblaciones de cohortes con muchos individuos durante periodos largos de tiempo, muestran una prevalencia de la litiasis en torno al 8\%, y han revelado el efecto favorecedor que para la formación de cálculos oxalocálcicos tiene la reducción del calcio de la dieta. Este hallazgo ha tenido un gran impacto entre los urólogos, pues ha supuesto un cambio notable en la práctica clínica. La restricción del contenido cálcico de la dieta, que clásicamente se recomendaba a los pacientes con litiasis cálcica, debe ser abandonada por el efecto negativo que tiene esta medida en los pacientes. Otras revelaciones interesantes de estos estudios han sido que el riesgo de formación de cálculos disminuye con un mayor consumo de líquidos y alto contenido de potasio en la dieta, y aumenta con un mayor consumo de proteínas de origen animal y de carbohidratos (aunque éste último factor solamente fue observado en el grupo de mujeres).

La evaluación de los factores de riesgo para cada paciente debe comenzar con una buena historia clínica (antecedentes familiares de urolitiasis, hábitos dietéticos, factores medio-ambientales, ingesta de fármacos, existencia de enfermedades sistémicas, etc.) y el estudio de composición del cálculo (evaluación de historia clínica y análisis el cálculo). Solamente los pacientes con especial riesgo litogénico deben ser sometidos a una evaluación más ampliada. Este estudio, conocido como estudio metabólico-mineral, se ha simplificado mucho en los últimos años de forma que es muy fácilmente realizable por la mayoría de los laboratorios. Se debe determinar el calcio, fósforo ácido úrico, sodio, cloro, potasio y creatinina en sangre y orina de 24 horas, también el oxalato, citrato y el magnesio en orina de 24 horas que, junto con la determinación de la diuresis diaria y el $\mathrm{pH}$ de orina, nos puede dar una estimación aproximada de la capacidad litogénica de la orina. Toda alteración morfológica y funcional del sistema excretor (malformaciones, dilatación, obstrucción, lesión tisular, etc.) debe ser considerada como potencial factor favorecedor de la litogénesis.

Las medidas de prevención generales (ingesta líquida, consejos dietéticos, modificación de hábitos de vida, etc.) son aconsejables en todos los pacientes. En aquellos que existan alteraciones metabólicas marcadas y con litiasis recurrente deben ser sometidos a tratamiento farmacológico selectivo (tiazidas en hipercalciuria, alopurinol en hiperuricemia, citrato potásico en hipocitraturia, etc.). Con estas simples medidas correctoras se consigue reducir la recidiva litiásica por debajo del $25 \%$ a largo plazo.

En conclusión, en los albores del siglo XXI el tratamiento integral de la litiasis implica el conocimiento y adiestramiento por parte del urólogo de varios procedimientos: la LEOC, la NLP, la URS, la laparoscopia y la cirugía abierta. La identificación y la posterior corrección de factores de riesgo litogénico consigue reducir considerablemente la posibilidad de recidiva litiásica. 